\title{
Improving Supragluteal Contouring Using Curved Liposuction Cannulas Introduced Through a Single Midline Port
}

\author{
MOHAMED ASHRAF, M.D.; AYMAN NOAMAN, M.D. and AHMED ELNAGGAR, M.D. \\ The Department of Plastic \& Reconstructive Surgery, Faculty of Medicine, Cairo University, Egypt
}

\begin{abstract}
Aim: The definition of beauty has changed in the last 1015 years where women seek more a highly defined curved body regarding buttocks in relation to the waist and thighs. The supragluteal area needs a long learning curve to have a smooth transition between the back and gluteal region. In our study we adopted a single port liposuction for this technically difficult region using a curved liposuction cannula instead of the usual three port site incisions and a straight cannula adopted by many surgeons.
\end{abstract}

Method: From 2017-2020, 94 Female patients had gluteal augmentation using fat grafting and liposuction of the lower trunk, age ranging from 22-54 with a mean age of 32. Studying the use of a single incision in the intergluteal crease for liposuction of the supragluteal area using the curved shaft liposuction cannula size $3 \mathrm{~mm}$ and size $4 \mathrm{~mm}$ to reach the desired contour instead of adding another two paramedian supragluteal incisions. The main goal is to reach the upper lateral supragluteal region.

Results: The transition zone between the lower back and buttocks was greatly achieved with a very high surgeon and patient satisfaction in more than $85 \%$ of the cases. ( 80 cases), There was no any major aesthetic complication in terms of Step-off's, irregularities, over-resection or musculanization.

Conclusion: Curved liposuction cannulas can greatly contribute to minimizing unnecessary extra incisions to reach various areas in the body specially the technically challenging upper outer supragluteal region as mentioned in our study.

Level of Evidence: Therapeutic, V.

Key Words: Liposuction - Buttocks augmentation - Fat grafting.

\section{INTRODUCTION}

The gluteal region is one of the most important aspect of beauty in almost all cultures, yet the definition of beauty has changed in the last 10-15 years where women seek more a highly defined curved body regarding buttocks and breast in relation the to the waist and thighs [1]. Gluteal beauty comes in its smooth curve from flanks to the knee and the softness of its fat and good skin quality $[2,3]$.
The concept of gluteal augmentation with fat grafting is all about re-distributing the fat from areas with excess to other areas which is in need aesthetically. Utilizing this concept, even buttocks with a big amount of fat usually appear aesthetically better and appears more contoured after adding fat. So, it is more liposculpture than enhancement. Consequently, patients with gross mal distribution of fat will benefit from the fat redistribution [4].

The purpose of gluteal fat grafting with 360 liposuction is to change the square or ptotic shape of buttocks-hip area to an A-type frame, narrowest at waist-to-hip ratio that gradually widens towards the hips [5].

Despite many papers discussing the gluteal liposculpture, there are very few studies tangling the incisions needed to achieve results or the type of cannulas specific for these areas regarding shafts and port shapes. Hoyos described single midline incision over intergluteal cleft for lower back liposculpture, and 1 infragluteal for fat grafing [6]. However, Mendieta described three incisions, one in midline above the intergluteal crease and two paramedian incisions in lower back for achieving adequate sculpture of the upper outer supragluteal area $[4,5]$.

\section{Anatomical considerations:}

According to Mendieta he categorized the buttocks into 4 main frames, A-shaped, V-shaped, Square shape and round shape. Yet there is always asymmetry and many patients who fall in-between 2 categories.

Mendieta defines the upper lateral area as zone 3 (Fig. 1), [7]. Hoyos markings for supragluteal area wasn't much different and he highlights that it's lower part is a very important area and any over-resection is extremely difficult to correct [6]. 
In our study we will evaluate the improvement in supergluteal contouring using curved liposuction cannulas introduced through a single midline port instead of the more common technique used of the 3 ports.

\section{MATERIAL AND METHODS}

From 2017-2020, 94 Female patients had gluteal augmentation using fat grafting and liposuction of the lower trunk, age ranging from 22-54 with a mean age of 32. Mean BMI was 29 ranging from 26-32.

Studying the use of a single incision in the intergluteal crease for liposuction of the supragluteal area using the Curved shaft liposuction cannula size $3 \mathrm{~mm}$ and size $4 \mathrm{~mm}$ to reach the desired contour instead of using adding another two paramedian supragluteal incisions.

We excluded patients with coagulopathies, high body mass index (greater than 32), or who've had previous surgery in the region.

\section{All Buttocks frames were included in our study:}

All patients were consented regarding the use of data and photographs taken before and after the procedure using the same photography technique as Wong et al., in 2016, and Heidekrueger et al., $[8,9]$.

\section{Pre-operative markings:}

We have found that Hoyos markings are anatomically easier to apply on all patients with a very specific landmark. We draw a line from the point of maximum indentation (PMI) in the flanks to the upper point of intergluteal crease. Another line from iliac crest to the previously marked intergluteal crease point. Above the Green line in the illustration where fat should be suctioned to the maximum in this area. The $\mathrm{C}$ point was identified which is the upper limit of the trochanteric depression and then trace the 3 rd line to the upper point of the intergluteal crease forming another triangle. This is the transitional zone between the waist and the hip which must have a smooth transition and any over-resection will end in poor aesthetic outcome. (Fig. 2).

\section{Technique:}

All our patients had full clinical examination and their fitness for surgery was assessed according to the American Society of Anaesthesiologists (ASA) grades I \& II criteria with Infiltration of the selected areas for liposuction was performed according to the Klien formula [10].
A single incision for infiltration and liposuction was done in intergluteal crease (the apex of the $\mathrm{V}$ sub-unit). No Port site protection ports were used due to lack of availability despite being useful. Another incision in infragluteal fold which was used only for gluteal fat injection.

The waistline area was suctioned deeply and thoroughly by $3 \mathrm{~mm}$, $4 \mathrm{~mm}$ curved cannula aiming at maximum waistline definition (Fig. 3). Liposuction over the lumbar region must be performed carefully to avoid trauma to the underlying structures or even penetration of the abdominal cavity.

Zone 3 according to Mendieta, which coincides with the upper lateral supragluteal area according to Hoyos would never be reached with a straight shaft cannula from the intergluteal crease incision only and any trials will end up with an undesired depression in the superior gluteal region which we need to augment further (Fig. 4).

A second run of liposuction was done in supragluteal region after fat injection. We had no drains in our study and the port site was closed with monocryl 4/0.

\section{Fat grafting:}

Fat tissue was harvested with the $3 \mathrm{~mm}$ and $4 \mathrm{~mm}$ blunt cannula to the sterile canister. Decantation was used to separate fat from saline and blood. Through the mid inferior gluteal incision which was the standard in our patients however in a few we needed to inject more fat from our intergluteal midline liposuction port, the injection cannula was inserted and the fat was inserted with a fanning motion from deep to superficial. Most of the graft is placed in the middle third over gluteus, with additional graft placed to upper or lower poles if needed. We also followed all recent recommendations for safe fat grafting, [11] with continuous motion during injection superficial placement of fat and not in the muscle and we avoided high graft fill pressure. The Volume injected ranged between $300-850 \mathrm{ml}$ per side.

It's not always easy to quantify the amount of fat to be injected in each patient and the site and the areas where we need to inject more depending on the shape of the buttocks where asymmetry is common. However, an A-shape buttock is usually improved with more liposuction in upper lateral and lower lateral buttocks with more fat grafting in the middle lateral depressed part. The square shaped will go with the same with less fat grafting in the lateral part and concentrating our fat in the middle. The V-shaped buttocks were difficult to 
contour but we concentrate on the lower lateral part which is greatly depressed in these patients. but almost all areas of buttocks are grafted concentrating mainly on the middle third.

Our end point of fat grafting was subjective according to the surgeon and the patient preference decided pre-operatively but we usually stop when the fat continuously comes out of our injection site to avoid high fill pressure. Infragluteal incision site for grafting was closed with monocryl 5/0.

\section{Post-operative care:}

Patients had their compression garments for 6 weeks post-operative. Early mobilization from the night of surgery. Garments were removed the day after surgery and manual drainage massage was done by a physiotherapist or by the surgeon to release extra fluid collection though the port sires. All patients were discharged the next day following surgery. We recommended minimizing pressure on the grafted fat in gluteal region for 3 weeks postoperative although there's no evidence in literature regarding this. Patients were instructed to visit at $3,7,15,25$ days post-operative and then bimonthly for 1 year.

\section{RESULTS}

The Mean follow-up of patients was 8 months, ranging from 5 months to 12 months. The transition zone between the lower back and buttocks was greatly achieved with a very high surgeon and patient satisfaction in 80 of our cases $(85 \%)$.

There was no step-off's or irregularities and a $\mathrm{C}$-shaped waist with a smooth transition to the buttocks and lateral thighs was achieved. Addition-

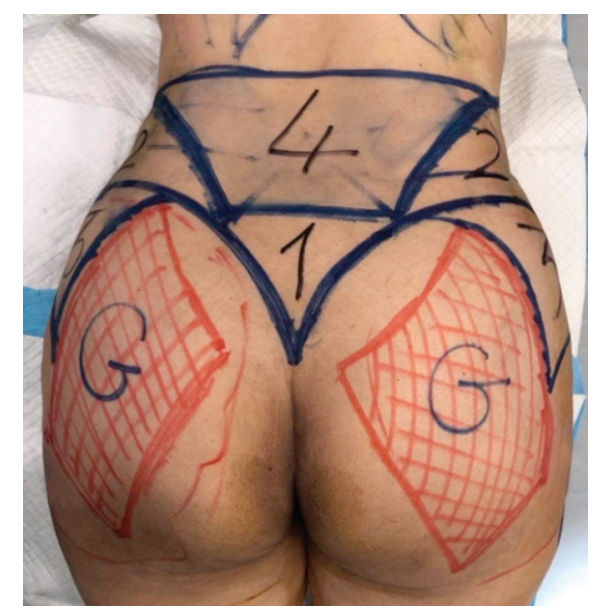

Fig. (1): Mendieta's Zones of liposuction "quoted from Mendieta" [6,5]. ally, no Masculinized buttocks, or over-resection was elicited.

There were no major complications in the term of skin necrosis, major infection, unfavorable results requiring re-operation, DVT, thromboembolism or fat embolism.

However, minor port site infection in the intergluteal incision occurred in 14 patients (15\%) which was managed conservatively with local ointments.

Seroma in the back occurred in $8(8.5 \%)$ patients which required multiple percutaneous aspirations

1 patient had patchy skin rash which might be related to fluid leak or the garment material which was changed to another type after the first postoperative visit and it resolved in a few days.

The patients were asked to grade their rate of satisfaction from their gluteal contouring specifically and not other areas of liposuction that wasn't mentioned in our study. Highly Satisfied, moderately satisfied, dissatisfied. Also if the dissatisfied patients require re-operation for aesthetic improvements (Table 1).

The the main reason for the dissatisfaction was the amount of graft amount 12 patients wanted a bigger size and projection, and 2 from the moderately satisfied group wanted a smaller size.

Table (1): Post-operative patient's satisfaction.

\begin{tabular}{lccc}
\hline $\begin{array}{l}\text { Highly } \\
\text { satisfied }\end{array}$ & $\begin{array}{c}\text { Moderately } \\
\text { satisfied }\end{array}$ & Dissatisfied & $\begin{array}{c}\text { Patient need } \\
\text { for re-operation }\end{array}$ \\
\hline 80 cases & 5 cases & 9 cases & $\begin{array}{l}\text { None } \\
0 \%\end{array}$ \\
$85.1 \%$ & $5.3 \%$ & $9.57 \%$ & 0 \\
\hline
\end{tabular}

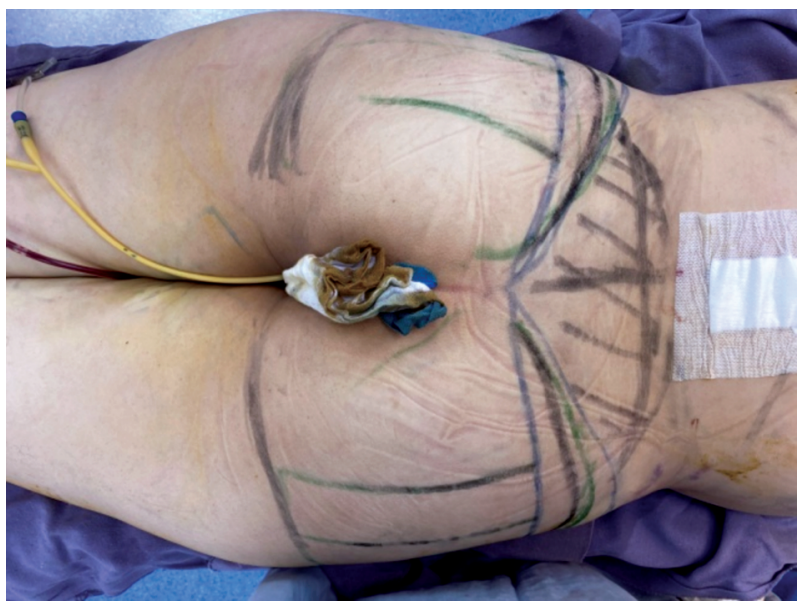

Fig. (2): Markings technique as described by Hoyos, where the supraglutealtraingle bas is the most critical [6]. 


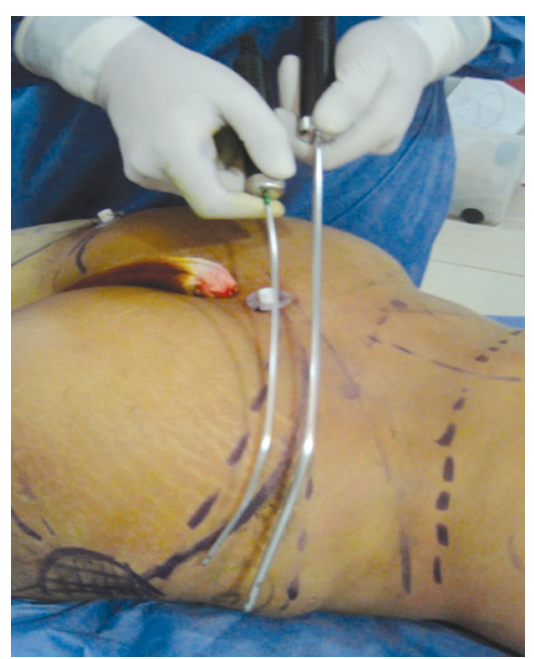

Fig. (3): Curved cannula reach to the upper lateral supragluteal region.

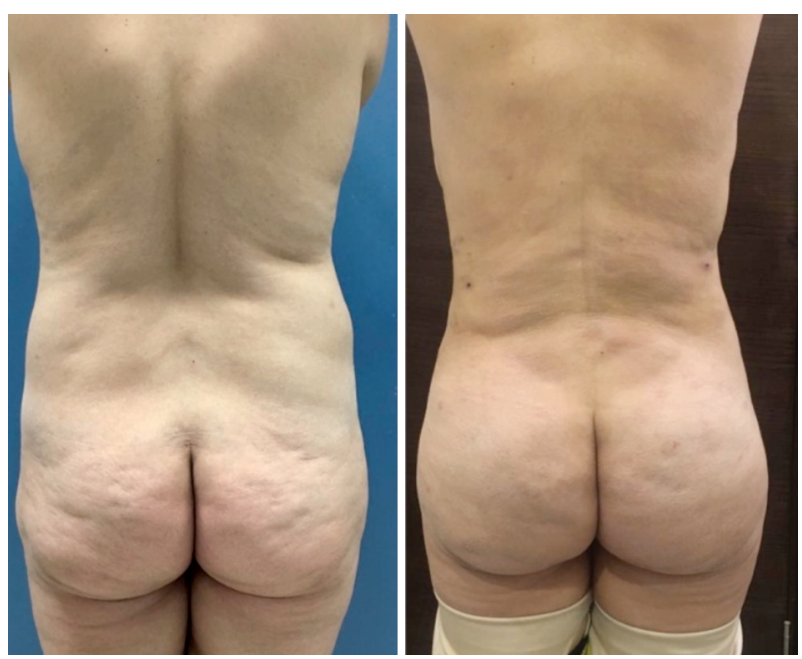

Case (1): Right before, left after, note the smooth transition from waist to hips.

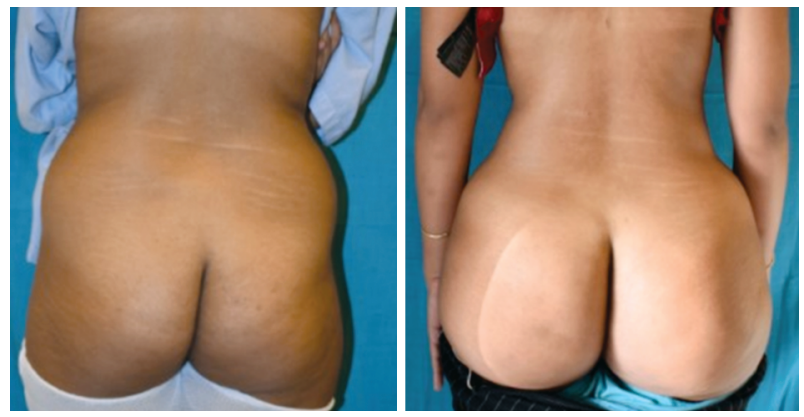

Case (3): Right - Before, Left - After; note the improvement.

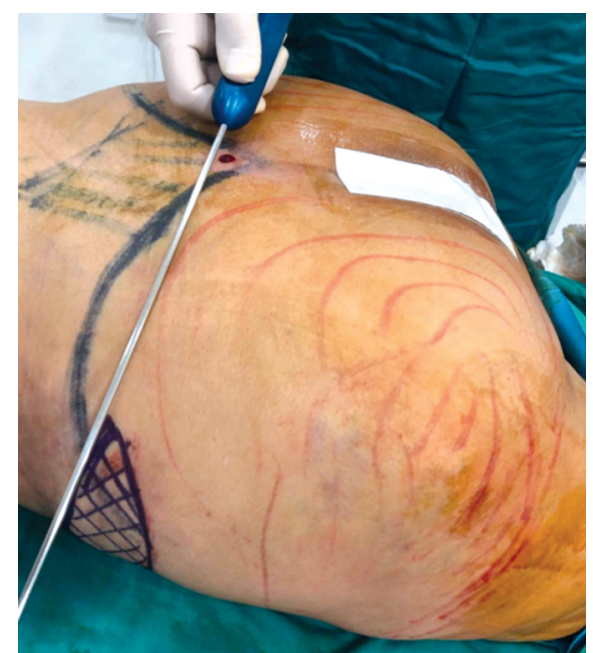

Fig. (4): Straight cannula can't reach unless it passess through buttocks proper.

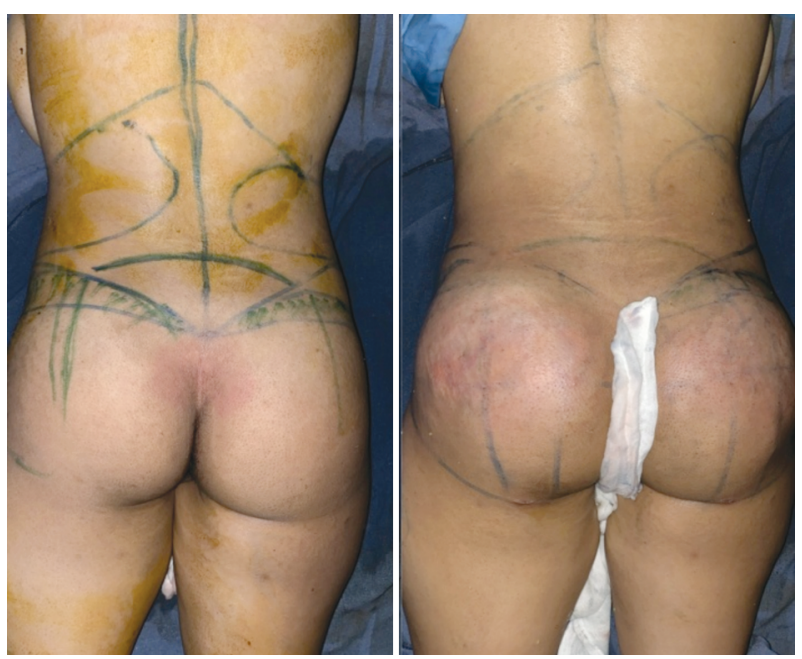

Case (2): Right - Before, Left - After.

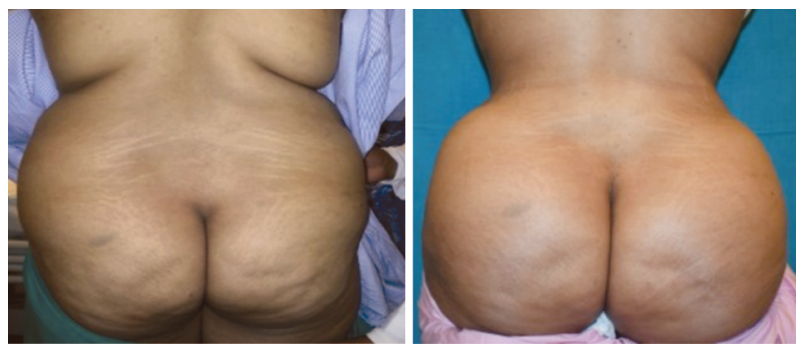

Case (4): Right - Before, Left - After.

Case (5): Right - Before, Left - After.
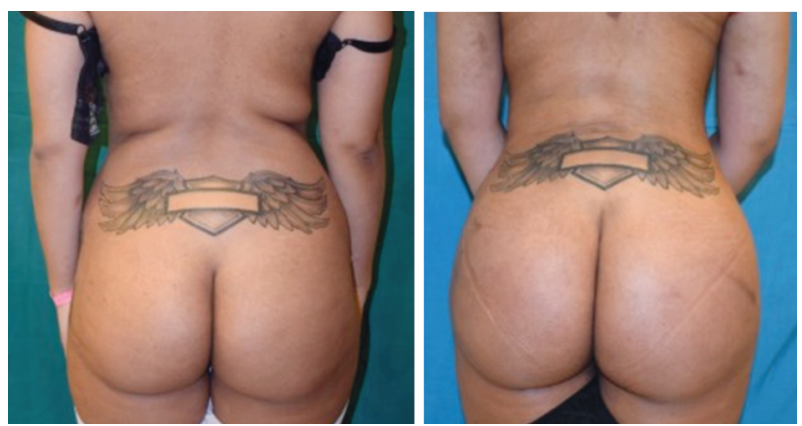


\section{DISCUSSION}

The literature is very scarce regarding the incisions for supragluteal liposuction and very deficient regarding cannula shaft shapes. Mendieta as one of the pioneers in this field, he had many concerns in his studies regarding this area. In 2007, he mentioned that he had some unsatisfying results like step-off's or square shaped buttocks after a couple of months despite having a good shape intraoperatively. Also, he suggested that the superolateral area as described by Hoyos is the most unforgiving zone in the buttocks that needs a long steep learning curve. His recommendations were as follow, one should decide how much fat to remove exactly from pre-operative photos, he added lateral decubitus position instead of the previous prone/supine only. Finally, he recommended cross hatching this area by adding the $2^{\text {nd }}$ incision at the superior posterior iliac spine [4]. In 2018, Mendieta didn't change these recommendations where he still used the 3 incisions for the supragluteal area or zone 3 according to his illustrations [5]. He also highlighted that more than $80 \%$ of the contouring procedure comes from the liposuction and that the fat grafting comes as an additional factor in buttocks enhancement [7]. Yet he never mentioned if he used any other than the straight cannulas.

Hoyosused $4.6 \mathrm{~mm}$ curved and $4.6 \mathrm{~mm}$ semicurved cannulas for waistline liposuction from the intergluteal incision only. Adding incisions in the exposed bare area of the back was done by him only in patients with really back rolls or secondary cases and in both cases it wasn't in the supragluteal area. He postulated that fat over-suctioning in this zone would end up masculinizing the buttocks and it'll be extremely difficult to correct where many fat grafting sessions would be needed and if the surgeon can't figure out the end-point of liposuction for this area leave more for a touch up instead of doing more harm with over-correction. Also, he confirmed that this area requires so much training and a long learning curve [6].

Kim et al., in a study of 2398 patients performing superficial liposuction had Seroma rate of $2.25 \%$ [12], Thomas, $\mathrm{M}$ et al., had a $3.5 \%$ [13]. Also Taha, A et al., had a seroma rate of $34 \%$ [14]. However none had really compared different areas of liposuction and the exact incidence of seroma in each. In our study we had $8.5 \%$ seroma incidence were we suspect the main reason is due to high mean BMI of our cases which correlates with Iverson, R.E \& Pao study mentioning that high BMI increases seromas significantly [15]. Cannula port site burn and infection incidence wasn't exactly mentioned in literature however it was mentioned that it is a relatively common complication where many authors have tried to decrease it with the use of different techniques and gadgets $[\mathbf{1 6 , 1 7 , 1 8 ]}$.

Villenueva used a curved cannula in injecting fat and says it helps the surgeon to remain in superficial planes. But he did not mention using it for liposuction or highlight the incisions needed for suctioning the waistline [11].

Beck et al., recently mentioned that the cannula is the most important and variable item in a liposuction procedure and despite its importance there is no yet universal standards or protocols in their design in ports and shafts with a very few studies contributing to the aid of any [19].

\section{Conclusion:}

Curved liposuction cannulas introduced through a single midline port improved supragluteal contouring, prevented step off deformities, excessive resection and prevented an supragluteal scars.

\section{REFERENCES}

1- Cuenca-Guerra R. and Quezada J.: What makes buttocks beautiful? A review and classification of the determinants of gluteal beauty and the surgical techniques to achieve them. Aesth. Plast. Surg., 28: 340-347, 2004.

2- Da Rocha R.P.: Surgical anatomy of the gluteal region's subcutaneous screen and its use in plastic surgery. Aesthetic Plast. Surg., 25: 140-144, 2001.

3- Bauccu O., Gozil R., Ozmen S., et al.: Gluteal region morphology: The effect of the weight gain and aging. Aesthetic Plast. Surg., 26: 130-133, 2002.

4- Mendieta C.: Gluteal reshaping. Aesthetic Surgery Journal, 27 (6): 641-655. doi:10.1016/j.asj.2007.09.001, 2007.

5- Mendieta C. and Stuzin J.M.: Gluteal Augmentation and Enhancement of the Female Silhouette. Plastic and Reconstructive Surgery, 141 (2): 306-311. doi:10.1097/ prs.0000000000004094, 2018.

6- Hoyos A.E. and Prendergast P.M.: High Definition Body Sculpting, doi:10.1007/978-3-642-54891-8, 2014.

7- Mendieta C.: Classifcation system for gluteal evaluation. Clin. Plast. Surg., 33: 333-346, 2006.

8- Wong W.W.1, Motakef S., Lin Y. and Gupta S.C.: Redefining the Ideal Buttocks: A Population Analysis Plast. Reconstr. Surg., 137 (6): 1739-47, 2016.

9- Heidekrueger P.I., Sinno S., Tanna N., Szpalski C., Juran S., Schmauss D., Ehrl D.Ng.R., Ninkovic M. and Broer P.N.: The Ideal Buttock Size: A Sociodemographic Morphometric Evaluation. Plast. Reconstr. Surg., 140 (1): 20e-32e, 2017.

10- Klien J.A.: The tumescent technique. Anesthesia and modified liposuction technique. Dermatol. Clin., 8 (3): 425-37, 1990.

11- Villanueva N.L., Del Vecchio D.A., Afrooz P.N., Carboy J.A. and Rohrich R.J.: Staying Safe during Gluteal Fat 
Transplantation. Plastic and Reconstructive Surgery, 141 (1): 79-86. doi:10.1097/prs.0000000000003934, 2018.

12- Kim Y.H., Cha S.M., Naidu S. and Hwang W.J.: Analysis of Postoperative Complications for Superficial Liposuction: A Review of 2398 Cases. Plastic and Reconstructive Surgery, 127 (2): 863-871. doi: 10.1097/prs.0b013e 318200 afbf, 2011.

13- Thomas M., Menon H. and D'Silva J.: Surgical complications of lipoplasty-management and preventive strategies. Journal of Plastic, Reconstructive \& Aesthetic Surgery, 63 (8): 1338-1343. doi:10.1016/j.bjps.2009.06.046, 2010.

14- Taha A.A., Wahba M.M. and Tahseen H.: Liposuction. Plastic and Reconstructive Surgery - Global Open, 8 (3): e2677. doi:10.1097/gox.0000000000002677, 2020.

15- Iverson R.E. and Pao V.S.: MOC-PS(SM) CME Article: Liposuction. Plastic and Reconstructive Surgery, 121
(Supplement): 1-11. doi: 10.1097/01.prs.0000308480. 33644.56, 2008.

16- Bank J. and Song D.H.: Inexpensive Method of Liposuction Cannula Port-Site Protection. Aesthetic Plastic Surgery, 37 (4): 843-843. doi: 10.1007/s00266-013-0130-4, 2013.

17- Cheryl Hui* and Christopher Chui: Liposuction cannula port site protection: An innovative strategy, JPRAS Open. Jun., 16: 81-83, 2018.

18- Khoo L.S., Corona G.G., Radwanski H., Fernandes V.S and Pitanguy I.H.: Inexpensive Method of Liposuction Cannula Port-Site Protection. Aesthetic Plastic Surgery, 38 (1): 256-257. doi: 10.1007/s00266-013-0241-y, 2013.

19- Beck D.O., Davis K. and Rohrich R.J.: Enhancing Lipoaspirate Efficiency by Altering Liposuction Cannula Design. Plastic and Reconstructive Surgery Global Open, 2 (10): e222. doi: 10.1097/gox.0000000000000101, 2014. 